\title{
Efecto relativo de la edad en el circuito profesional de Pádel The relative age effect in the professional Padel circuit
}

\author{
Alejandro M uñoz, Carla Serrano-González, Iñaki Q uintana_García, Miriam Granado-Peinado \\ Universidad Francisco deVitoria (España)
}

\begin{abstract}
Resumen: El pádel ha pasado a ser a uno de los deportes más practicados en España. El propósito de este estudio fue evaluar el Efecto Relativo de la Edad (RAE) en los jugadores del circuito deW orld Pádel Tour (W PT) ( $n=395)$ teniendo en cuenta el mes de nacimiento, género, nacionalidad, edad y ranking. La distribución trimestral obser vada no fue significativamente diferente $(X ; p>.05)$ a la esperada para el grupo general o para cual quiera de las subcategorías analizadas. Por tanto, no parece confirmarse un efecto de la edad relativa en los jugadores profesionales de pádel analizados, considerando el ranking, género y nacionalidad, a diferencia de lo que ocurre en otros deportes de raqueta. En este sentido, esta investigación constata que la fecha de nacimiento no es un factor relevante en el circuito deW PT y confirma que llegar a la élite del pádel no se basa exclusivamente en factores físicos y antropométricos.
\end{abstract}

Palabras clave: Efecto relativo de la edad; Maduración biológica; Selección de talento; Edad cronológica; Pádel.

\begin{abstract}
Padel has become one of the most practiced sports in Spain. The purpose of this study was to evaluate the Relative Effect of Age (RAE) in theWorld Padel Tour (W PT) circuit players $(n=395)$ taking into account the month of birth, gender, national ity, age and ranking The quarterly distribution observed was not significantly different $\left(X^{2} ; p>.05\right)$ than expected for the general group or for any of the subcategories analyzed. Ther efore, it does not seem to confirm an effect of relative age on professional padel players analyzed, considering the ranking, gender and nationality, unlike what happens in other racket sports. Thus, this investigation confirms that the date of birth is not a relevant factor in theW PT circuit and confirms that reaching the elite of the paddle that is not based exclusively on physical and anthropometric factors.
\end{abstract}

Keywords: Relative age effect; Biological maturation; Tal ent identification; Chronological age; Padel.

\section{Introducción}

El pádel es un deporte de carácter intermitente, de colaboración-oposición que se encuentra dentro del grupo de los deportes de raqueta y se juega por parejas, dos contra dos (Sánchez-Alcaraz, Courel-Ibáñez y Ca ñas, 2017; Martínez, 2013). Este deporte, popular entre los países de habla hispana, ha pasado a ser uno de los más practicados en España (CSD, 2019). Según la encuesta de hábitos deportivos en España (M ECD, 2015), el porcentaje de personas que practican este deporte semanalmente pasó de un 1,7\% en 2010 a un 3,7\% en 2015, aumentando el número de licencias federadas de 49. 463 en 2014 a 72.266 en 2018. En un estudio llevado a cabo por Courel-Ibáñez, Sánchez-Alcaraz, GarcíaBenítez y Echegaray (2017) estimaron 4,2 millones de practicantes de pádel en 2015, aumentando esta cantidad en 3,0 millones de personas respecto al año 2010. Este crecimiento podría estar en relación con las bajas demandas fisiológicas que implica este deporte en comparación con otros de raqueta, así como el hecho de

\footnotetext{
Fecha recepción: 03-12-19. Fecha de aceptación: 13-01-21

Alejandro Muñoz Moreno

alejandro.munoz@ufv.es
}

jugarse en un campo de juego reducido y contener reglas simples (Lasaga, 2011; Sánchez-Alcaraz y SánchezPay, 2014).

El efecto relativo de la edad (RAE) hace referencia a la diferencia de edad cronológica entre los integrantes de un mismo grupo deedad (Campo, 2013), lo que puede acentuar diferencias psicológicas y físicas relacionadas con el mes de nacimiento de las personas (Andronikos, Elumaro, Westbury y Martindale, 2015). Ello puede conllevar consecuencias de carácter físico, cognitivo, de experiencia o de maduración por el simple hecho de haber nacido en el primer o último trimestre del año (Campo, 2013; Redd, Fukuda, Beyer y O liviera, 2018). Uno de los primeros estudios en el que se evaluó y comprobó el efecto relativo de la edad sobre el rendimiento académico en estudiantes, fue llevado a cabo por Dickinson y Larson (1963). Específicamente, en el ámbito deportivo, uno de los estudios pioneros en el que se investigaron las diferencias y consecuencias de este efecto se realizó en jugadores de hockey sobre hielo y voleibol de origen canadiense, los cuales competían a nivel recreativo, senior y profesional (Grondin, Deschaies y Nault, 1984). Los resultados de este estudio mostraron una mayor frecuencia de jugadores de hockey en el primer trimestre de nacimiento en las 3 
categorías mencionadas, mientras que en voleibol estos resultados solo se observaron a nivel profesional.

A lo largo de los últimos años, este efecto ha sido ampliamente estudiado en numerosos deportes como el baloncesto (García, Vergara, Pérez y Cabezos, 2018), balonmano (W rang et al., 2018), atletismo (BrazoSayavera, M artínez-Valencia, M üller, Andronikos y Martindale, 2018), triatlón (O rtigosa-M árquez, Reigal, Serpa y Hernández-M endo, 2018; Ferriz, Sellés, García y Cejuela, 2020), voleibol (Angioluci, Reeberg, Nunes, Benetti y Pellegrinott, 2016) o fútbol, siendo este último donde mayor relevancia ha adquirido al mostrar una representación significativa de jugadores nacidos a principio del año (Bidaurrazaga-Letonaet al., 2015). En el tenis, deporte emparentado con el pádel, también ha sido analizado este efecto en jugadores jóvenes (BaxterJones, 1995; Dudink, 1994; Edgar y O 'D onoghue2005; Giacomini, 1999) aportando una gran influenciadel RAE sobre el rendimiento. Sin embargo, se observan resultados no tan favorables, sobre todo en muestra femenina (Giacomini, 1999; Ryan, 1989). Un reciente estudio de Gerdin, Hedberg y Hageskog (2018) realizado en jugadores de tenis suecos nacidos entre 1998 y 2001 concluyó que los mejores jugadores habían nacido en la primera mitad del año. Esta influencia ofrece más oportunidades a los que desde edades tempranas destacan por su condición física por el simple hecho de haber nacido a principios de año y tener un desarrollo físico, madurativo y cognitivo superior (W iium, Lie, O mmundsen y Enksen, 2010). A su vez, conlleva que aquellos que son elegidos aumentan su volumen de entrenamiento o práctica deportiva, ampliando además la frecuencia en la que compiten, generándose así una dinámica que ofrece todavía más ventajas respecto a los no seleccionados en un principio (Ericsson, Krampe y Tesch-Römer, 1993; Baker y Horton, 2004). No obstante, existen deportes como el surf (Redd et al. , 2018) donde el RAE no parece existir, y otros deportes como el fútbol sala en el cuál este efecto se produce de manera contraria (Lagos-Fuentes, Rey, Padrón-Cabo, Prieto-Troncoso y García-N úñez, 2020).

Como consecuencia de lo anteriormente menciona do y la falta de estudios hasta la fecha en una muestra de deportistas de pádel, resulta interesante analizar si existe efecto relativo de la edad en los jugadores de pádel del circuito de W PT. Específicamente, se plantea el comprobar si existen diferencias en el efecto relativo de la edad en función del género (hombres o mujeres), de la nacionalidad (española, argentina u otras), del ranking que ocupan en el circuitoW PT (desde el top 10 hasta el top 200) y del rango de edad (14-24; 25-35; 36-46; $45-$ 57).

\section{Método}

\section{Muestra}

La muestra estuvo conformada por los 395 jugadores y jugadoras del circuito W PT, de los cuáles se recogieron las fechas de nacimiento, así como información acerca de la nacionalidad, género, la posición en el ranking y la edad. La muestra quedó distribuida por género en 196 jugadores $(49,6 \%$ ) y 199 jugadoras $(50,4 \%$ ); por nacionalidad en 277 jugadores españoles $(70,1 \%)$, 66 argentinos (16,7\%) y 52 de otras nacionalidades $(13,2 \%)$; y por rango de edad en 165 jugadores entre los 14 y 24 años (41,8\%), 182 jugadores entre los 25 y 35 años (46,1\%), 45 jugadores entre los 36 y 46 años $(11,4 \%)$ y 3 jugadores entre los 45 y 57 años $(0,7 \%)$.

\section{Procedimiento}

A través de la página web del circuito W PT (www. worldpadeltour.com), se recopiló la información de todos los jugadores y jugadoras participantes durante el mes de diciembre de 2018, coincidiendo con el fin de temporada. Tras la búsqueda y recogida de datos, se elaboró una base de datos con la información de interés de los jugadores y jugadoras del circuito W PT (género, nacionalidad, ranking y edad). La muestra fue clasifica da en Q1 (enero-marzo), Q2 (abril-junio), Q 3 (julioseptiembre) y Q 4 (octubre-diciembre), en función del trimestre de nacimiento.

\section{Análisis de Datos}

Los datos fueron analizados con el programa estadístico SPSS 22.0. Para establecer las diferencias en la distribución de los porcentajes de jugadores en cada trimestre de nacimiento, se realizaron pruebas de ChiCuadrado $\left(X^{2}\right)$, estableciendo la significación estadística en $\alpha=.05$, una para cada análisis realizado del efecto relativo de la edad, en el total de jugadores y jugadoras en función del género; según la nacionalidad, del rango de edad, y, por último, en función del ranking. Además, se llevó a cabo una prueba de Correlación de Spearman para comprobar si existía relación entre el cuartil de nacimiento y el ranking de los jugadores y jugadoras.

\section{Resultados}

A continuación, se muestran los resultados obtenidos de la muestra estudiada. En la figura 1 se observa la 
distribución de los 395 jugadores y jugadoras del circuito de W PT, distribuidos por trimestres en cuatro cuartiles (Q 1, Q2, Q 3 y Q 4) según las fechas de nacimiento. Los resultados evidencian, a nivel descriptivo, una cier ta homogeneidad en la distribución de los nacidos en los tres últimos trimestres, siendo el primero donde menos nacidos se encuentran, no mostrándose diferencias estadísticamente significativas $(p=.470)$.

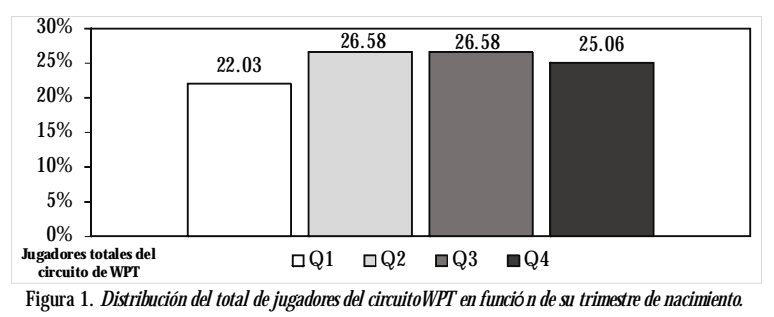

En relación con la distribución de los nacimientos en función del género (ver figura 2), los resultados revelan que tampoco existen diferencias estadísticamente significativas en el trimestre de nacimiento $(p=.612)$. Por otra parte, los análisis realizados con los jugadores, masculinos y femeninos por separado, tampoco muestran diferencias estadísticamente significativas para los jugadores $(p=.325)$ ni para las jugadoras $(p=.961)$.

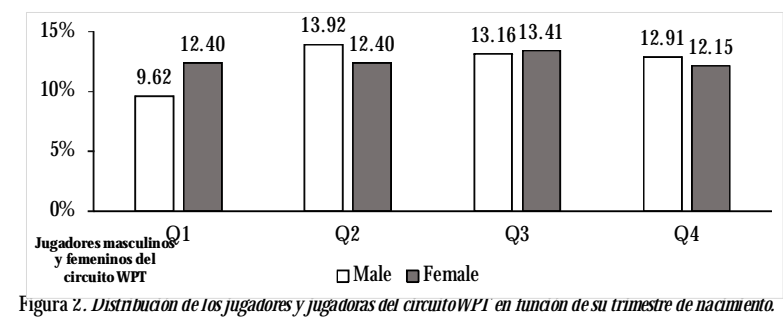

En la tabla 1 se presentan los descriptivos y pruebas estadísticas realizadas para el análisis de la distribución de los cuartiles de nacimientos según la nacionalidad. Los resultados informan de que no hay diferencias estadísticamente significativas entre los trimestres, en función de la nacionalidad española, argentina u otras $(p=.277)$.

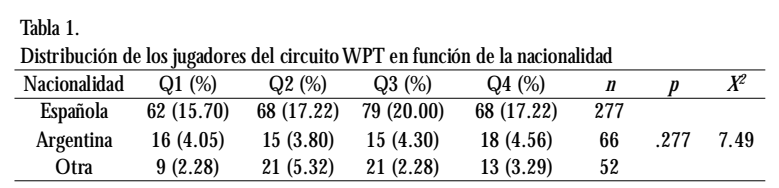

Siguiendo con el análisis del efecto relativo de la edad en función de la variable de rango de edad (ver tabla 2), los resultados ponen de manifiesto que no existen diferencias estadísticamente significativas $(p=.245)$ entre los trimestres según los cuatro rangos de edad dispuestos.

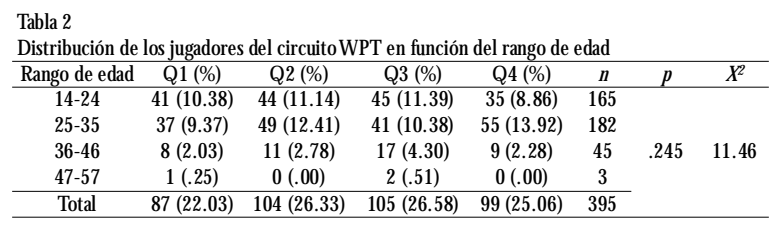

Por último, en la tabla 3 se detallan los resultados de los trimestres de nacimiento de los jugadores deW PT en relación al ranking, segmentando la muestra de 10 en 10. De nuevo no se encontraron relaciones significa tivas entre los cuartiles de nacimiento de los jugadores y su posición en el ranking $(p=.831)$, habiendo una distribución homogénea de los mismos. Tampoco se encontraron diferencias estadísticamente significativas en los cuartiles de nacimiento en función del ranking cuando éste se segmentó en grupo de $10(p=.708)$ ni dentro de cada agrupación (115. > p > 867).

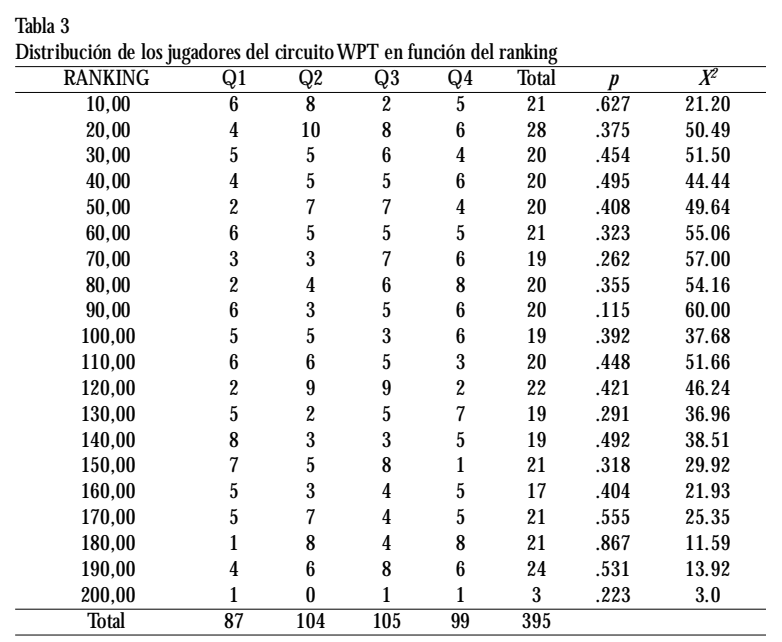

En resumen, los análisis realizados indican que no existe efecto relativo de la edad en el circuito World Padel Tour, independientemente de las variables analizadas (género, nacionalidad, rango de edad y ranking).

\section{Discusión}

El objetivo del presente estudio fue estudiar el efecto relativo de la edad en jugadores de pádel del circuito World Padel Tour. Los resultados no mostraron evidencias de este efecto en la muestra de deportistas analizada. Al igual que encontraron Redd et al. (2018) cuando anal izaron el cuartil de nacimiento de surfistas, nuestro estudio manifiesta que no existe un efecto relativo de la edad en los jugadores de pádel del circuito W PT, ni en el total de jugadores ni diferenciándolos por género, nacionalidad, rango de edad o posición en el ranking. Este resultado podría explicarse por el nivel de 
profesionalización de los distintos deportes. El pádel y el surf, aunque no han sido considerados deportes ma yoritarios en el pasado, están adquiriendo mayor relevancia nacional e internacional, así como mayor representación en los últimos años. Prueba de ello es la reciente incorporación del surf en los próximos juegos 0 límpicos de J apón 2020. Este hecho puede conllevar que no exista un proceso de captación de talentos tan desarrollado como lo hay, por ejemplo, en el fútbol (W iium et al., 2010). De modo que cabría esperar que, si estos deportes continúan en auge y adquieren cada vez más importancia dentro del mundo deportivo, y más practicantes en diversos países, los procesos de selección se refinasen y se captaran jugadores desde eda des más tempranas, lo que facilitaría la aparición del efecto relativo de la edad en deportes como el pádel dentro de unos años.

No obstante, y en contraposición con los resultados obtenidos, la literatura en otros deportes como como baloncesto (García et al., 2018), balonmano (W rang et al., 2018), atletismo (Brazo-Sayavera et al., 2018), triatlón (O rtigosa-Márquez et al., 2018), voleibol (Angioluci et al., 2016) o tenis (Gerdin et al., 2018) sí se ha demostrado un efecto positivo cuando se trata de deportistas de categorías inferiores. Asimismo, este efecto también se encuentra en categorías profesiona les en deportes como fútbol (González-Víllora, PastorVicedo y Cordente, 2015; Salinero, Pérez, Burillo, Lesma y Herrero, 2014; Salinero, Pérez y Lesma, 2013), ba Ioncesto (Esteva, Drobnic, Puigdellivol, Serratosa y Chamorro, 2006) o balonmano (Sánchéz,Y áñez, Sillero y RivillaGarcía, 2012).

Una posible explicación a la inexistencia del efecto relativo de la edad en el presente estudio podría encontrarse en las características físicas y antropométricas que precisan cada uno de estos deportes. Por ejemplo, en el proceso de captación de talentos de jugadores de baloncesto la altura, la velocidad o la agilidad han sido consideradas variables críticas (H oare, 1999); en el caso del fútbol, han sido relevantes la edad esquelética o la composición corporal (N orikazu, 2009). Sin embargo, en pádel, variables antropométricas como la altura, el peso 0 el índice de masa corporal no parecen ser tan relevantes como lo son para otros deportes de raqueta como el tenis o el bádminton (Sánchez-M uñoz, Sanz y Zabala, 2007; Pradas de la Fuente, González-Jurado, García-Giménez, Gallego Tobón, y Castellar 0 tín, 2019).

Estos hallazgos podrían dirigir futuras líneas de investigación a analizar el RAE en jugadores profesiona les de pádel de categorías inferiores. En la línea de los estudios mencionados, es posible que el RAE desaparezca o se reduzca a medida que aumenta la edad de los deportistas.

Esta investigación nos sirve de impulso para seguir analizado en RAE en deportes minoritarios o con menor profesionalización y en diferentes categorías, con el fin de comprobar si efectivamente este efecto solo se hace visible cuando la selección de los jugadores conlleva una mayor exigencia a nivel físico madurativo y en edades inferiores.

\section{Conclusiones}

Este estudio es el primero, hasta donde al canzanuestro conocimiento, que anal iza el RAE en jugadores de pádel, en concreto, jugadores del circuito profesional W PT. Los resultados obtenidos no apoyan la existencia del efecto relativo de la edad en este deporte.

Se hace necesario seguir investigando en futuros estudios sobre este efecto, ya que el pádel, además de ser un deporte relativamente joven, en los últimos años se está expandiendo por diferentes continentes, por lo que es esperable un crecimiento en participación, profesionalización y un mejor proceso de detección de talentos.

\section{Referencias}

Andronikos, G., Elumaro,A. I.,Westbury,T. \& Martindale, R. (2016). Efecto delaedad relativa: implicaciones paralaprácticaefectiva, Journal of Sports Sciences, 34(12), 1124-1131.

Angiolud, F., Reeberg, L.C., Nunes, F., Benetti, L.C.\& Pellegrinott, I. L. (2016). The Relative Age Effect in Male Volleyball Championships. International Journal of SportsScience, 6(3), 116120.

Baker, J. \& Horton, S. (2004). A review of primary and secondary influences on sport expertise. High Ability Studies, 15(2), 21128.

Baxter-Jones,A., Helms, P., M affull, N., Baines-Preece, J., \& Preece, M. (1995). Growth and development of male gymnasts, swimmers, soccer and tennis players: A longitudinal study. Annals of H uman Biology, 22, 381-394.

BidaurrazagazLetona, I., Badiola, A., Granados, C., Lekue, J. A., Amado, M. , \& Gil, S. M. (2015). Efecto relativo delaedad en futbol: estudio en un clubVasco profesional. Retos. Nuevastendencias de Educación Física, Deportey Recreación, 25, 95-99.

Brazo-Sayavera, ., Martínez-Valencia, M.A., Müller, L.,Andronikos, G. \& Martindale, R. J. J. (2018). Relative age effects in international age group championships: A study of Spanish track and field athletes. Plos One, 13 (4), e0196386.

Campo, D. G. (2013). Revisión y propuestas de intervención so- 
breel Efeecto de la Edad Relativaen losámbitos educativo y deportivo. Retos: Nuevas tendencias de Educación Física, Deportey Recreación, 23, 51-63.

Courel-Ibáñez, J., Sánchez-Alcaraz, B. J., García-Benítez, S., \& Echegaray, M. (2017). Evolución del pádel en Españaen función del género y edad de los practicantes. Cultura, Ciencia y Deporte, 12(34), 39-46.

Dickinson, D. J. , \& Larson, J. D. (1963). Effects of chronological agein monthson school achievement. Thejournal of Educational Research, 56(9), 492- 493.

Dudink, A. (1994). Birth date and sporting success. Nature, 368(6472), 592.

Edgar, S., \& O 'D onoghue, P. (2005). Season of birth distribution of elite tennis players. Journal of Sports Science, 23(10), 10131020.

Ericsson K. A., Krampe, R.T. \&Tesch-Römer, C. (1993).Therole of deliberate practice in the acquisition of expert performance. Psychology Review, 100 (3), 363-406.

Esteva, S., Drobnic, F., Puigdellivol, J., Serratosa, L. \& Chamorro, M. (2006). Date of birth and successin professional basketba II. Apunts, 41(149), 25-30.

Ferriz, A. , Sellés, S. , García, M. \& Cejuela, R. (2019). Efecto de la edad relativaparael desarrollo del talento en jóvenestriatletas. Retos: Nuevas tendencias de Educación Físca, Deportey Recreación, $37(37), 27-32$.

García,J., Vergara, J. M., Pérez,A. M. \& Cabezos, H. (2018). Efecto de la Edad Relativa de los Jugadores de Baloncesto en los Campeonatos del Mundo Sub17 y Sub19 y en los Juegos O límpicos 2016. Entrenamiento deportivo, 32(3).

Gerdin, G., Hedberg, M. \& Hageskog, C. (2018). RelativeAge Effect in Swedish Male and Female Tennis Players Born in 1998-2001. Sports (Base),6(2), 38.

Giacomini, C. P. (1999). Association of birthdate with success of nationally ranked junior tennis players in the United States. Perceptual and motor skills, 89(2), 381-386.

González-Víllora, S. , Pastor-Vicedo, J. C. \& Cordente, D. (2015). RelativeAge Effect in UEFA Championship Soccer Players. Journal of H uman Kinetics, 47, 237-248.

Grondin, S., Deschaies, P. \& N ault, L. P. (1984)Trimesters of birth and school output. Apprent Social, 1, 169-74.

Hoare, D. G. (1999). Predicting success in junior elite basketball players: the contribution of anthropometric and physiological attributes Journal of Science and Medicine in Sport, 3(4), 391405.

Lago-Fuentes, C., Rey, E., Padrón-Cabo, A., Prieto-Troncoso, J., \& Garcia-Núñez, J. (2020). The Relative Age Effect in Professional Futsal Players. Journal of human kinetics, 72, 173183.

Lasaga, M. J. (2011). Estudio social y metodológico del pádel desde la percepción de técnicas y jugadores una apuesta educativa (Tesis doctoral). Universidad deSevilla, Sevilla.

Martínez, B. J. A. (2013). Historia del pádel. Materiales para la historia del deporte, 11, 57-60.

Ministerio de Educación, Cultura y Deporte (2015). Encuesta de
HábitosD eportivosen España. M adrid: SecretaríaGeneralT écniCaMECD.

Norikazu, H. (2009). Relationships among birth-month distribution, skeletal age and anthropometric characteristicsin adolescent elitesoccer players. Journal of SportsSciences 27(11), 1159-1166.

O rtigosa-Márquez, J. M., Reiga, R., Serpa, S. \& Hernández-M endo, A. (2018). Efectos de laedad relativa en el proceso de selección nacional de triatletas. Revista Internacional de Medicina y Ciencias de la Actividad Físca y del Deporte, 18 (70), 199-211.

Pradas de laFuente, F., González-Jurado, J.A., García-Giménez, A., GallegoTobón, F., \& Castellar O tín, C. (2019). Característicasantropométricas, dejugadores de pádel deélite. Estudio piloto. Revisa Internacional de Mediana y Ciencias de La Actividad Físca y Del Deporte, 19(74), 181-195.

Redd, M.J., Fukuda, D. H., Beyer, K. S. \& O liviera, L. P. (2018). No O bservable Relative Age Effects in Professional Surfers: a Constraints-Based Evaluation. International Journal of Exercise Science, 11(6), 355-363.

Ryan, P. (1989). The redative age effect on minor sport participation (Tesis Doctoral). McGill University, Montreal, Q uebec.

Salinero, J.J., Pérez, B., Burillo, P., Lesma, M. L. \& Herrero, M. H. (2014). Efecto de edad relativaen el fútbol profesional espa ñol. Revista Internacional de Medicina y Ciencias de la Actividad Físca y el Deporte, 14(56), 591-601.

Salinero,J.. J., Pérez, B. , Burillo, P. \& Lesma, M. L. (2013). Relative ageeffectineuropean professional football.Analysisby position. Journal of Human Sport and Exercise, 8(4), 966-973.

Sánchez-Alcaraz, B. J., Courel-Ibáñez, J., \& Cañas, J. (2017). Estructura temporal, movimientos en pistay acciones de juego en pádel: revisión sistemática. Retos. N uevastendenciasdeEducación Física, Deportey Recreación, 33, 308-312.

Sánchez-M uñoz, C., Sanz, D., \& Zabala, M . (2007).Anthropometric characteristics, body composition and somatotype of elite junior tennis players. British Journal of Sports Medicine, 41(11), 793-799.

Sánchéz, C., Yáñez, A., Sillero, M . \& Rivilla-García, J. (2012). El efecto relativo de la edad en el balonmano de élite masculino en España. E-balonmano. Revista de Ciencias del Deporte, 8(3), 181-190.

Sánchez-Alcaraz, B. \& Sánchez-Pay,A. (2014). Medición delacondición física del jugador de pádel a través de tests. TRAN CES, 45-62.

W iium, N., Lie, S. A., O mmundsen,Y. \& H. R. Enksen (2010). DoesRelativeAgeEffect Existamong Norwegian Professional Soccer Players?. International Journal of Applied Sports Sciences, 22(2), 66-76.

W rang, C. Rossing, N., Diernæs, R. M., Hansen, C. G., DalgaardHansen, C. \& Karbing, D. S. (2018). RelativeAge Effect and the ReSelection of Danish MaleH andball Playersfor National Teams. Journal of Human Kinetics, 31(63), 33-41.

Zabala, L. J., Gil, S. M., Badiola, A., Bidaurrazaga, L. I.,Vargas,A., \& Granados, C. (2016). Característicasfísicasyantropométricas, y análisis de juego en jugadores de pádel de elite. Búsqueda, 3(16), 33-40. 